\title{
Time-Resolved sub-Ångström Metrology by Temporal Phase Interferometry near X-Ray Resonances of Nuclei
}

\author{
Stephan Goerttler®, ${ }^{1}$ Kilian Heeg, ${ }^{1}$ Andreas Kaldun, ${ }^{1}$ Patrick Reiser, ${ }^{1}$ Cornelius Strohm, ${ }^{2}$ Johann Haber, ${ }^{2}$ \\ Christian Ott, ${ }^{1}$ Rajagopalan Subramanian, ${ }^{1}$ Ralf Röhlsberger, ${ }^{2,3}$ Jörg Evers, ${ }^{1}$ and Thomas Pfeifer ${ }^{1}$ \\ ${ }^{1}$ Max-Planck-Institut für Kernphysik, 69117 Heidelberg, Germany \\ ${ }^{2}$ Deutsches Elektronen-Synchrotron DESY, 22607 Hamburg, Germany \\ ${ }^{3}$ The Hamburg Center for Ultrafast Imaging, Luruper Chaussee 149, 22761 Hamburg, Germany
}

(Received 21 March 2019; published 10 October 2019)

\begin{abstract}
We introduce an analytical phase-reconstruction principle that retrieves atomic scale motion via timedomain interferometry. The approach is based on a resonant interaction with high-frequency light and does not require temporal resolution on the time scale of the resonance period. It is thus applicable to hard $\mathrm{x}$ rays and $\gamma$ rays for measurements of extremely small spatial displacements or relative-frequency changes. Here, it is applied to retrieve the temporal phase of a $14.4 \mathrm{keV}$ emission line of an ${ }^{57} \mathrm{Fe}$ sample, which corresponds to a spatial translation of this sample. The small wavelength of this transition $(\lambda=0.86 \AA)$ allows for determining the motion of the emitter on sub-Ångström length and nanosecond timescales.
\end{abstract}

DOI: 10.1103/PhysRevLett.123.153902

The measurement of tiny translations and motional patterns is central to a large array of questions in fundamental science (e.g., quantum physics [1-4] and gravitational-wave detection [5]) as well as technological applications (e.g., precision-engineering and material processing). A common approach is interferometry using coherent optical (laser) light, based on the constructive and destructive interference of waves [1,2]. By relating two different points in space, time, momentum or energy, interferometry translates a range of measurements into the common problem of determining phase differences. In addition, interferometry has led to breakthroughs in precision metrology since the seminal work of Ramsey [6].

Retrieving the phase information is also at the heart of quantum dynamics, where the motion of wave packets is given by the relative phases between participating states. Time-resolved spectroscopy techniques aim at extracting these relative phases between quantum states by sequences of short pulses of light [7], and thus getting experimental access and control of the underlying quantum motion. In this context, the method of spectral interferometry was developed $[8,9]$ to reconstruct the relative spectral phase of two pulses, i.e., electromagnetic wave packets. This fueled the rise of optical coherence tomography [10] and other interferometry techniques, which find applications in chemical [11] and biomedical [12] areas of research. Because of the visible to XUV light sources used thus far, the spatial resolution in such applications has been limited to few nanometers [13] up to now.

In this Letter, we further develop the concept of temporal phase interferometry $[14,15]$, which allows for the extraction of the relative temporal phase of emitters in the time domain. The method is an inverse approach to spectral phase interferometry and establishes a heterodyne-type measurement concept without the need of a temporally coherent local oscillator. This in particular suggests applications at the highest frequencies ( $\mathrm{x}$ or $\gamma$ rays), where such reference oscillators thus far do not exist. The key requirement for the applicability of the method is the availability of resonances in the light-matter interaction, which are sufficiently narrow such that their decay can be observed directly in the time domain. Similarly, the phase changes itself must be slow enough to be directly detectable. However, there is no constraint on the cycle period of the light itself, provided that the associated photons can be detected. After introducing the method with a toy model, we demonstrate it in the hard $\mathrm{x}$-ray domain employing Mössbauer spectroscopy to directly retrieve sub-atomicscale displacements with nanosecond temporal resolution.

To introduce the scheme, we consider a toy model with two single-line emitters, separated spectrally by a frequency detuning $\Delta$. One of the emission lines exhibits an explicit time-dependent phase $\varphi(t)$ [Fig. 1(c)] relative to the other [see Fig. 1(a)]. The detuning causes an interference pattern in the time domain [Fig. 1(b)], which is manipulated by the temporal phase $\varphi(t)$. The idea here is to directly retrieve this phase from the Fourier transform of the measured time- and energy-dependent interferogram.

The resonance frequencies of emitter 1 and emitter 2 are given by $\omega_{0}$ and $\omega_{0}+\Delta$, respectively, while the linewidth of both emitters is given by $\gamma$. Emitter 1 is additionally modified by the time-dependent phase $\varphi(t)$ [Fig. 1(c)], e.g., by transient motional Doppler shifts or Stark shifts for the case of atoms. In the time domain, the electric fields of the two decaying resonance emitters can classically be described by exponentially decaying dipole responses. 


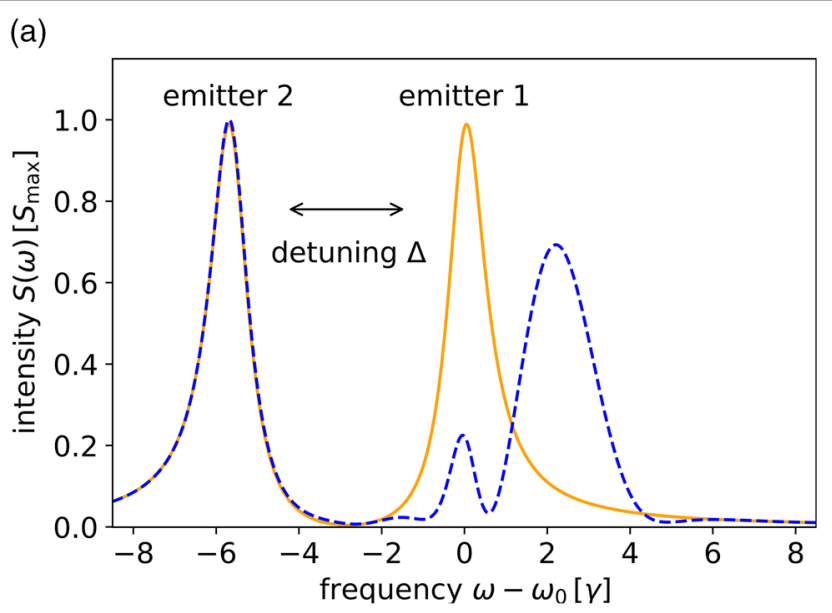

(b)

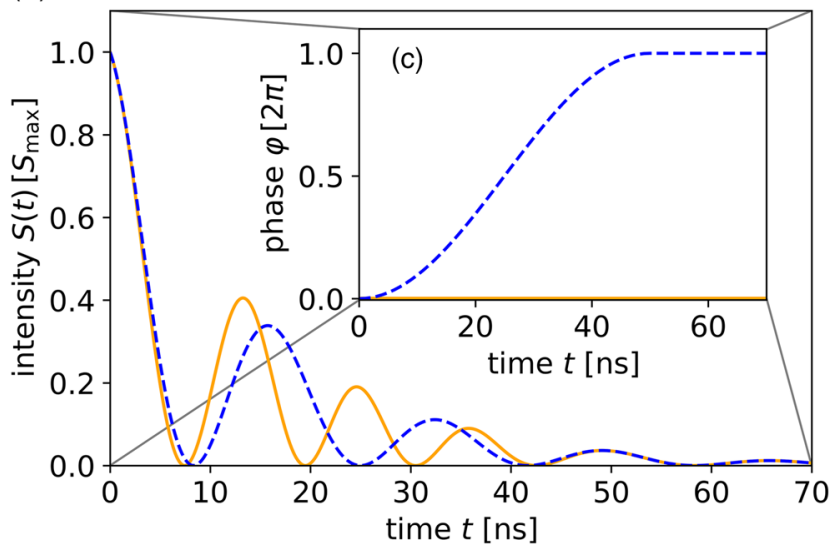

FIG. 1. Spectral (a) and temporal (b) intensity of two single-line emitters separated by a frequency detuning $\Delta<0$. In both panels, the orange solid line corresponds to a temporally constant phase between the two emitters, whereas the blue dashed line illustrates the case of a temporally varying phase shown in (c). For the temporally constant phase, the two emission frequencies lead to a beating in the temporal signal. The temporally varying phase leads to modulations of the temporal signal, and in turn imprints additional interference structures onto the spectrum.

The Heaviside step function $\theta$ models the onset of these dipole responses:

$$
\begin{aligned}
& E_{\mathrm{E} 1}(t) \propto-e^{i \varphi(t)} e^{-(\gamma / 2) t-i \omega_{0} t} \theta(t), \\
& E_{\mathrm{E} 2}(t) \propto-e^{-i \Delta \cdot t} e^{-(\gamma / 2) t-i \omega_{0} t} \theta(t) .
\end{aligned}
$$

This yields for the measurable time- and frequencydetuning-dependent interference signal, consisting of the sum of the two fields, the following expression:

$$
\begin{aligned}
S(t, \Delta) \propto & \left|E_{\mathrm{E} 1}(t)+E_{\mathrm{E} 2}(t)\right|^{2} \theta\left(t-t_{0}\right) \\
\propto & 2\{1+\cos [\varphi(t)+\Delta \cdot t]\} e^{-\gamma t} \theta\left(t-t_{0}\right) \\
= & 2 e^{-\gamma t} \theta\left(t-t_{0}\right)+e^{i[\varphi(t)+\Delta \cdot t]} e^{-\gamma t} \theta\left(t-t_{0}\right) \\
& +e^{-i[\varphi(t)+\Delta \cdot t]} e^{-\gamma t} \theta\left(t-t_{0}\right) .
\end{aligned}
$$
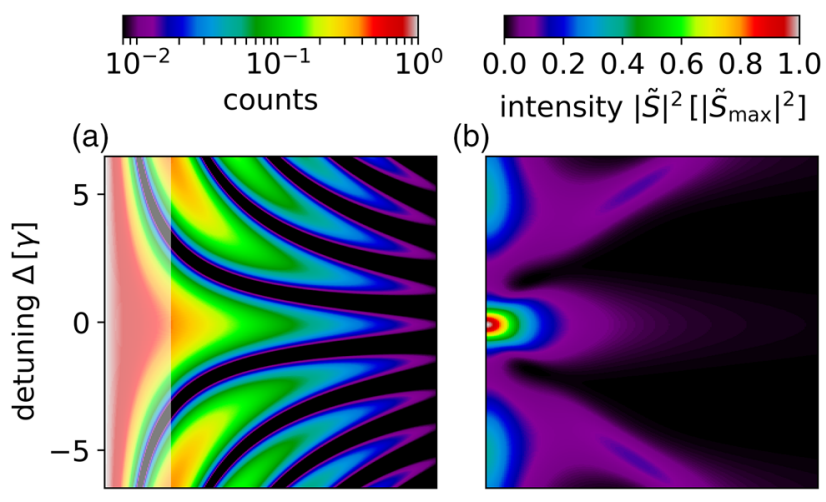

(b)
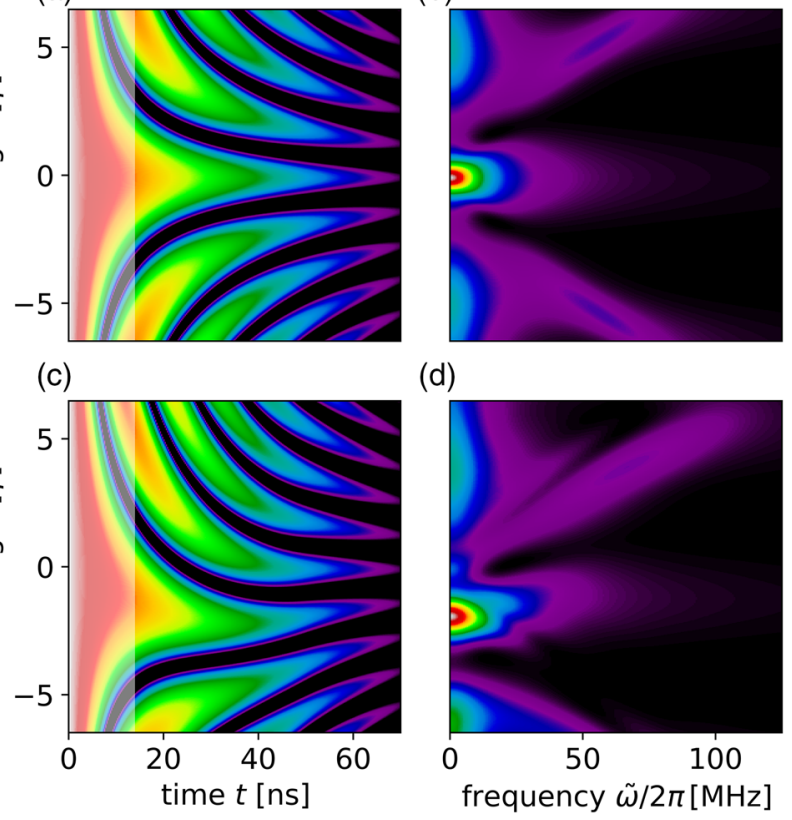

(d)

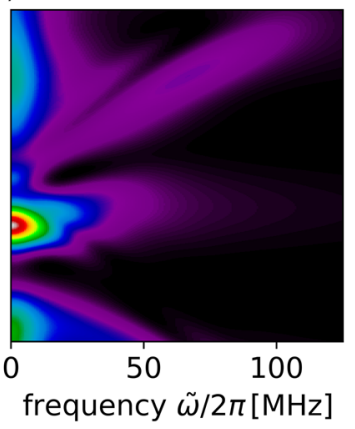

FIG. 2. (a),(c) Spectrogram $S(t, \Delta)$ as a function of time and frequency detuning with temporal phase $\varphi=0$ (a) and $\varphi=\varphi(t)$ (c) [see Fig. 1(c)]. The temporal phase modifies the resonance of the emitter and accounts for the asymmetry. (b),(d) The respective squared absolute Fourier-signal $|\tilde{S}(\tilde{\omega}, \Delta)|^{2}$ of (a) and (c), respectively. Values below $14 \mathrm{~ns}$ (transparent) were excluded from the Fourier transform to match the experiment in Fig. 3. The temporal phase $\varphi(t)$ causes a modulation of the positive oscillating term in (d), while the linear component of $\varphi(t)$ in the range of 20 to $40 \mathrm{~ns}$ corresponds to a detuning shift in (c). For high enough detuning, the nonoscillating and oscillating terms are separated, enabling a clear reconstruction of the phase evolution.

Here, the parameter $t_{0} \geq 0 \mathrm{~ns}$ is introduced to include experimental constraints as discussed below. The signal $S(t)$ and its respective phase $\varphi(t)$ are shown in Figs. 1(b) and 1(c) for $t_{0}=0$ ns and fixed $\Delta<0$. Figures 2(a) and 2(c) show the signal $S(t, \Delta)$ for $\varphi=0$ and $\varphi=\varphi(t)$, respectively.

The first step of the phase-reconstruction method is to Fourier transform the signal $S(t, \Delta)$ in Eq. (2) into the frequency domain to yield the Fourier-signal $\tilde{S}(\tilde{\omega}, \Delta) \propto \mathcal{F}\{S(t, \Delta)\}$. The absolute square of $\tilde{S}(\tilde{\omega}, \Delta)$ is shown in Figs. 2(b) and 2(d) for $\varphi=0$ and $\varphi=\varphi(t)$. To allow for a comparison with the experimentally obtained data, we set $t_{0}=14 \mathrm{~ns}$. In the second step, the nonoscillating term $\mathcal{F}\left\{2 e^{-\gamma t} \theta\left(t-t_{0}\right)\right\}$ is determined, for example, by recording each temporal decay of the emission lines separately, and then subtracted from the Fourier-signal $\tilde{S}(\tilde{\omega}, \Delta)$. Finally, a Fourier transform back to the time domain of the positive oscillating term $\mathcal{F}\left\{e^{i(\varphi(t)+\Delta \cdot t)} e^{-\gamma t} \theta\left(t-t_{0}\right)\right\}$, which is sufficiently separated from the negative oscillating term at high enough detuning 
$\Delta$, directly yields the phase $\varphi(t)+\Delta \cdot t$ for $t \geq t_{0}$ as the argument of the resulting complex-valued function of time. To determine the detuning $\Delta$ in terms of $\gamma$, if not known directly, a reference spectrogram with $\varphi(t)=0$, as shown in Fig. 2(a), can be used.

We performed our experiment at the Nuclear Resonance Beamline ID18 at ESRF in Grenoble [16], which provided $14.4 \mathrm{keV}$ x-ray pulses temporally separated by $176 \mathrm{~ns}$. In the experiment, each $\mathrm{x}$-ray pulse coherently excites ${ }^{57} \mathrm{Fe}$ nuclear ensembles in two consecutive stainless steel foils, which can each be regarded as single-line emitters of photons in the forward direction. The first foil ("emitter") contains $55 \%$ iron, which is enriched in ${ }^{57} \mathrm{Fe}$ to a large fraction, and is sputtered with a thickness of $d \approx 200 \mathrm{~nm}$ onto a piezoelectric transducer, or piezo. We can translate the emitter in both directions along the beam direction by applying a voltage $U(t)$ to the piezo (see also Refs. [17-20]). This translation $x(t)$ of the emitter corresponds linearly to the temporal phase $\varphi(t)=(2 \pi / \lambda) x(t)$ of the single-line emission. The piezo translation was electrically triggered and locked to the arrival time of the synchrotron pulses to achieve reproducibility. The second foil ("analyzer") consists of a ${ }^{57} \mathrm{Fe}$ single-line absorber and is moved with a constant velocity to Doppler shift it from the resonance with a frequency detuning $\Delta$. In the limiting description of thin absorbers, the emission lines for emitter and analyzer can be described by Eqs. (1a) and (1b), whereas the measurable interference signal is given by Eq. (2).

We measured the flux of scattered photons in forward direction with a stack of avalanche photodiodes with nanosecond temporal resolution. The detector is set to reject the first $14 \mathrm{~ns}$ after the arrival of the synchrotron pulse in order to omit the signal caused by the several orders of magnitude more intense prompt synchrotron pulse, which we used to excite the two emitters. Around 100000 photon counts were acquired for one spectrogram. Two measured spectrograms are depicted in Fig. 3, one without (a) and one with piezo translation (c). The bin size is $2 \mathrm{~ns} \times 0.18 \gamma$. The discrete Fourier transforms of the spectrograms are shown in Figs. 3(b) and 3(d). The similarity with the data computed within our toy model (see Fig. 2) is clearly visible.

We performed the above-described reconstruction for six spectrograms corresponding to different voltages to retrieve the temporal phase and therefore the translation of the emitter. This translation is a function of time relative to the arrival time of the synchrotron pulse. We used only data with $\Delta \gtrsim 3.0 \gamma(\Delta \lesssim-3.0 \gamma$ if $\Delta U<0)$ in order to separate more clearly the relevant phase information from the nonoscillating term in the Fourier-signal [Fig. 3(d)], as indicated by the horizontal lines in Figs. 3(c) and 3(d). Spectrograms with much reduced voltage $\Delta U \approx 0$, such as the one shown in Fig. 3(a), were used to determine the nonoscillatory component at $\Delta \approx 0$. Next, we subtracted the nonoscillatory component from the Fourier-signal. Most
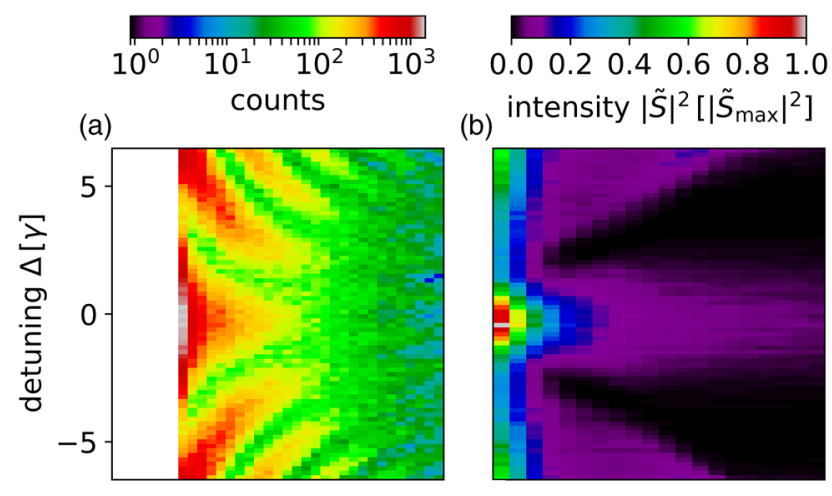

(b)

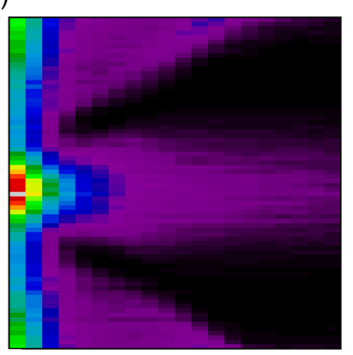

(c)

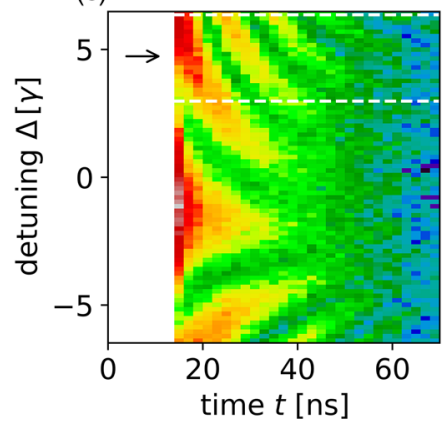

(d)

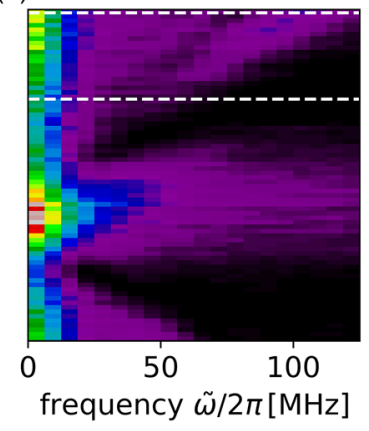

FIG. 3. (a),(c) Experimental spectrogram measured vs time and frequency detuning without (a) and with PT translation (c). Panels (b) and (d) depict the absolute square of the corresponding Fourier signals $|\tilde{S}(\tilde{\omega}, \Delta)|^{2}$. We used spectrogram (a) to calibrate the measurements. Only signals arriving after $t_{0}=14 \mathrm{~ns}$ are recorded in order to avoid saturation and dead time caused by the prompt synchrotron pulse at $t=0 \mathrm{~ns}$ which we used to excite emitter and analyzer. The phase reconstructed from the indicated area $\Delta \gtrsim 3.0 \gamma$ in (c) is shown in Fig. 4(a) (green).

likely due to different sample characteristics of the piezo and analyzer, the signal around $\tilde{\omega}=0$ does not approach zero, such that we manually set the value at $\tilde{\omega}=0$ to zero. Note that due to the large $\Delta$ this does not influence the desired positive oscillatory component. Afterwards, we Fourier transformed the positive frequencies back to the temporal domain, where we read out the complex phases for both the spectrogram with $|\Delta U|>0$ and the reference spectrogram with $\Delta U \approx 0$ to determine the temporal phase as the difference between the two. Lastly, we averaged the temporal phase over the detuning. The initial phase offset was determined by fitting Eq. (2) with $t=t_{0}=14 \mathrm{~ns}$ to each spectrogram. The statistical error of the phase is composed of the standard error of the mean and the standard error of the phase offset.

The results are presented in Fig. 4. The temporal phases in blue were realized by applying a voltage step of $\Delta U \approx$ $\pm 3.5 \mathrm{~V}$ to the piezo, whereas for the temporal phases in green a step of $\Delta U \approx \pm 7 \mathrm{~V}$ was applied (see Supplemental Material, Sec. I [21]). Consistently, the reconstructed phase-shift appears doubled for the latter case. Mounting the piezo onto a Plexiglas substrate to avoid mechanic 


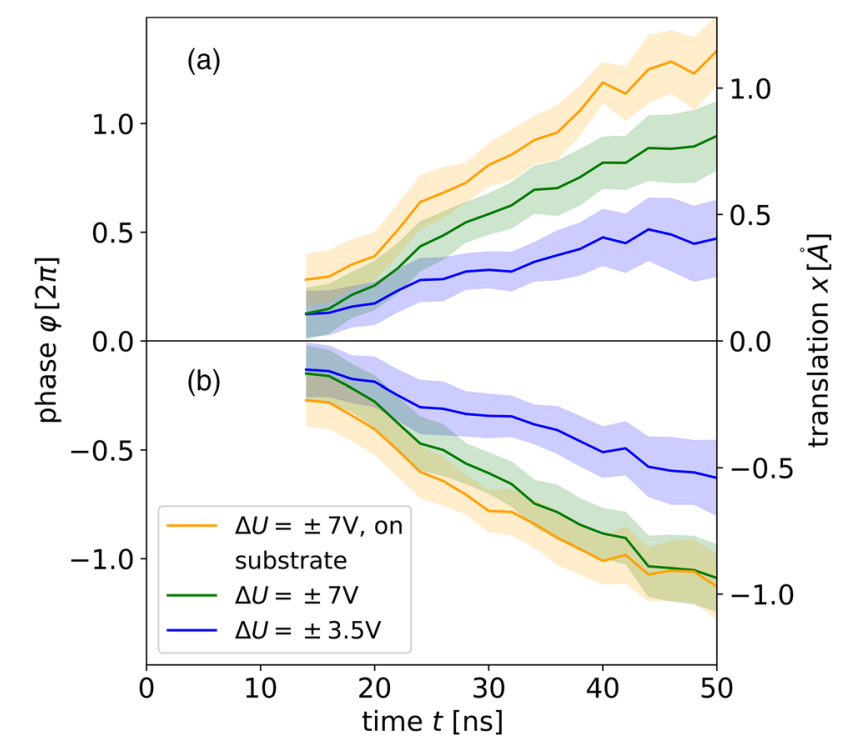

FIG. 4. (a),(b) Temporal phase and translation of the emitter retrieved from three measurements for (a) contraction $(\Delta U>0)$ and (b) extension $(\Delta U<0)$ of the piezo onto which the emitter is sputtered. The green phase in (a) was retrieved from the spectrogram shown in Fig. 3(c). The error intervals represent the statistical error discussed in the text.

perturbations further increased the temporal phase (orange), reflecting the displacive motion of the absorber foil. The approximate mirror symmetry between the temporal phases implies that the translations caused by piezo contraction [Fig. 4(a)] and extension [Fig. 4(b)] agree in magnitude. Statistical errors, illustrated as error intervals in Fig. 4, are small compared to the magnitude of the phase change.

The phase shifts can be straightforwardly converted into a length scale $x(t)=(\lambda / 2 \pi) \varphi(t)$ by using the Mössbauer resonance photon energy at $14.4 \mathrm{keV}$, which corresponds to a wavelength $\lambda=0.86 \AA$. This length scale is also given by the right vertical axis in Fig. 4. Accordingly, we retrieved piezo motions on a sub-Ångström length scale with nanosecond temporal resolution. The results are consistent with the estimated PT translations of $\Delta x \approx \pm 1.2$ and $\Delta x \approx \pm 0.6 \AA$ for voltage steps of $\Delta U= \pm 7$ and $\Delta U= \pm 3.5 \mathrm{~V}$, respectively (see Supplemental Material, Sec. II [21]).

In conclusion, we introduced a temporal interferometry concept that allows for a direct and fast retrieval of temporal phase shifts. The scheme is viable at any frequency and relies solely on the availability of a resonance for which the temporal decay must be resolvable in time. This was shown here exemplarily for the case of a Mössbauer nuclear transition in ${ }^{57} \mathrm{Fe}$. Note that a different method to reconstruct such displacements was already employed in Ref. [20]. However, it was based on a fully numerical approach in order to accommodate for samples with more complex individual responses. In contrast, the method developed here allows for a direct semianalytical reconstruction of the temporal phase, which is much faster and also provides a deeper understanding of the involved physics.

The direct metrology of small phase shifts at high photon energies allows to further optimize x-ray quantum-optical control methods, such as negative absorption on a resonance by mechanical motion of a resonant target [20]. In future developments, applying the method to even higherenergy gamma resonances of far-apart test masses, similar measurements of small relative phase shifts may allow for new ways of detecting gravitational waves.

We acknowledge funding from the European Research Council (ERC) (X-MuSiC-616783).

[1] T. Young, Phil. Trans. R. Soc. London 92, 12 (1802).

[2] C. J. Davisson and L. H. Germer, Proc. Natl. Acad. Sci. U.S.A. 14, 317 (1928).

[3] W. S. Warren, H. Rabitz, and M. Dahleh, Science 259, 1581 (1993).

[4] K. Hornberger, S. Gerlich, P. Haslinger, S. Nimmrichter, and M. Arndt, Rev. Mod. Phys. 84, 157 (2012).

[5] B. P. Abbott et al., Phys. Rev. Lett. 116, 061102 (2016).

[6] N. F. Ramsey, Phys. Rev. 78, 695 (1950).

[7] N. F. Scherer, R. J. Carlson, A. Matro, M. Du, A. J. Ruggiero, V. Romero-Rochin, J. A. Cina, G. R. Fleming, and S. A. Rice, J. Chem. Phys. 95, 1487 (1991).

[8] L. Lepetit, G. Chériaux, and M. Joffre, J. Opt. Soc. Am. B 12, 2467 (1995).

[9] E. Tokunaga, A. Terasaki, and T. Kobayashi, Opt. Lett. 17, 1131 (1992).

[10] D. Huang et al., Science 254, 1178 (1991).

[11] T. Masubuchi, H. Furutani, H. Fukumura, and H. Masuhara, J. Phys. Chem. B 105, 2518 (2001).

[12] B. P. Payne, V. Venugopalan, B. B. Mikić, and N.S. Nishioka, J. Biomed. Opt. 8, 273 (2003).

[13] S. Fuchs et al., Sci. Rep. 6, 20658 (2016).

[14] W. Sturhahn, Phys. Rev. B 63, 094105 (2001).

[15] R. Callens, C. L'abbé, J. Meersschaut, I. Serdons, W. Sturhahn, and T.S. Toellner, Phys. Rev. B 72, 081402 (2005).

[16] R. Rüffer and A. I. Chumakov, Hyperfine Interact. 97/98, 589 (1996).

[17] P. Schindelmann, Ph.D. thesis, Technische Universität München, 1999.

[18] U. van Bürck, W. Potzel, P. Schindelmann, G. Smirnov, S. L. Popov, E. Gerdau, Y. V. Shvyd'ko, H. D. Rüter, and O. Leupold, Hyperfine Interact. 141/142, 151 (2002).

[19] F. Vagizov, V. Antonov, Y. V. Radeonychev, R. N. Shakhmuratov, and O. Kocharovskaya, Nature (London) 508, 80 (2014).

[20] K. P. Heeg et al., Science 357, 375 (2017).

[21] See Supplemental Material at http://link.aps.org/ supplemental/10.1103/PhysRevLett.123.153902 for signal sequences applied to the piezoelectric transducer and for an estimation of the piezoelectric transducer translations. 